Research Article

\title{
The Diagnostic Yields and Safety of Double-Balloon Enteroscopy in Obscure Gastrointestinal Bleeding and Incomplete Small Bowel Obstruction: Comparison between the Adults and Elderly
}

\author{
Lei Wang $\mathbb{D}^{\mathbb{C}}$, Mengfan Xie, Liwen Hong, Chen Zhang, Tianyu Zhang $\mathbb{D}$, Rong Fan $\mathbb{D}$, \\ Jie Zhong $(\mathbb{D}$, and Zhengting Wang
}

Department of Gastroenterology, Ruijin Hospital, Shanghai, Jiaotong University School of Medicine, Shanghai, China

Correspondence should be addressed to Jie Zhong; jimmyzj64@hotmail.com and Zhengting Wang; zhengtingwang@shsmu.edu.cn

Received 11 March 2020; Accepted 20 April 2020; Published 27 April 2020

Academic Editor: Tatsuya Toyokawa

Copyright (C) 2020 Lei Wang et al. This is an open access article distributed under the Creative Commons Attribution License, which permits unrestricted use, distribution, and reproduction in any medium, provided the original work is properly cited.

Background. Double-balloon enteroscopy (DBE) is widely used worldwide. However, comparisons between the diagnostic yields in adults and the elderly remain scarce. Aim. The aim of this study is to compare the diagnostic yields and safety of DBE between adults and elderly with obscure gastrointestinal bleeding and incomplete small bowel obstruction. Method. We retrospectively reviewed patients who underwent DBE with indication of obscure gastrointestinal bleeding or incomplete small bowel obstruction in Ruijin Hospital and classified them into adults (18-64 years old) and elderly ( $\geq 65$ years old). Clinical characteristics, diagnostic yields, and postoperative complications were collected and further analyzed. Results. A total of 877 DBE procedures, 729 in adults and 148 in the elderly, were performed. In the patients with OGIB, the adults showed a higher frequency of Meckel's diverticulum compared with the elderly ( $4.6 \%$ vs. $0.9 \%, P=0.032)$. Angioectasia was higher in frequency in the elderly than in the adults $(25.9 \%$ vs. $17.9 \%, P=0.048)$. In patients with incomplete small bowel obstruction, the elderly were more likely to have adenocarcinoma than the adults $(19.4 \%$ vs. $7.1 \%, P=0.038)$. The adults had higher tendency to have Crohn's disease than the elderly $(23.4 \%$ vs. $8.3 \%, P=0.045)$. Most of the postoperative complications were mild. The adults and elderly displayed comparable tolerance to DBE $(P>0.05)$ Conclusion. DBE has a high diagnostic yield in small bowel disorders, and a slight difference in disease spectrum was observed between the adults and elderly. DBE can be well-tolerated in the elderly.

\section{Introduction}

Double-balloon enteroscopy (DBE) was first introduced by Yamamoto et al. in 2001 [1]. Since then, DBE has revolutionized the clinical approach in diagnosing, sampling, and treating small bowel diseases. Obscure gastrointestinal bleeding (OGIB), which has $69.8 \%-80.6 \%$ diagnostic yield, remains the most common indication for DBE $[2,3]$. Other indications for DBE include incomplete small bowel obstruction, abdominal pain, diarrhea, small bowel tumors, and pancreaticobiliary disorders in patients with surgically altered anatomy [4-6]. We previously demonstrated that the indications of OGIB and incomplete small bowel obstruction have the highest diagnostic yields [3]. However, despite its useful- ness, DBE is limited by some challenges. Compared with esophagogastroduodenoscopy (EGD), colonoscopy, and video capsule endoscopy (VCE), DBE is more technically complex and requires prolonged duration for endoscope insertion deep into the intestine, and thus, its invasiveness usually increases [7-9]. Therefore, DBE may be recommended to be feasible followed by conventional EGD, colonoscopy, VCE, and CT enterography [10].

Despite its long duration, DBE is a relatively safe procedure with a complication rate comparable to that of conventional endoscopic procedures [11-13]. However, clinicians are disinclined to use DBE in the elderly because it potentially increases risks associated with sedation, comorbidities, and relatively worsened cardiopulmonary function. Data on the 
use of DBE in elderly and between adults and elderly are limited [14-16]. In this study, we retrospectively compared the diagnostic yields and tolerance between adults and elderly patients who underwent DBE in our center.

\section{Method}

2.1. Patients Enrolled. A single-center retrospective study was designed. The medical records of $\geq 18$-year-old patients who underwent DBE with the indication of OGIB or incomplete small bowel obstruction from March 2008 to July 2019 at the Department of Gastroenterology, Ruijin Hospital, Shanghai, China, were reviewed. All of the enrolled patients were divided into two groups by their age (adults: 18-64 years old; elderly: $\geq 65$ years old). Patients with comorbidities of mild-to-moderate chronic diseases, such as hypertension and diabetes mellitus, were included in the analysis. Patients with OGIB had confirmed GI bleeding that is with a symptom of hematochezia or melena or lower hemoglobin plus occult blood test positive with exclusion of non-GI disease. Moreover, they had undergone upper or lower endoscopy and gained no positive findings. This study was approved by the Institutional Ethics Board of Ruijin Hospital.

2.2. Preoperative Preparation and DBE Procedure. Written informed consents were acquired from all patients preoperatively. The patients were required to intake a liquid diet with low residue at least 3 days from the procedure. Bowel cleansing was conducted the day before DBE procedure by using at least $3.0 \mathrm{~L}$ of a compound laxative agent with $132 \mathrm{~g}$ of polyethyleneglycol as the major component.

DBE (EN-450 P5/20, Fujifilm, China) was performed by three endoscopists (JZ, SC, and LW). The DBE system consists of a $200 \mathrm{~cm}$ long endoscope with a $140 \mathrm{~cm}$ overtube. The endoscope and overtube were equipped with an inflatable balloon at their distal tips. The bowel could be anchored through alternatively inflating the balloon of the endoscope or overtube. Thus, the endoscope could be inserted deep into the intestine.

The route of DBE procedure (antegrade or retrograde) was determined under the guidance of clinical symptoms and diagnostic tools. Patients who underwent both routes of the procedure were regarded to have underwent two separate DBEs. Conscious sedation with midazolam and fentanyl was induced in some patients during the DBE procedure.

2.3. Data Collection. Patients' gender, duration of symptom, past GI surgery, DBE time, route, and length of the examined intestine were recorded as clinical characteristics. The diagnostic yields of DBE with the indication of OGIB, including normal, adenocarcinoma, lymphoma, GISTs, Meckel's diverticulum, polyps, Crohn's disease, intestinal tuberculosis, Behcet's disease, angioectasia, and nonspecific enteritis, were retrieved. The diagnostic yields of DBE with the indication of incomplete small bowel obstruction, including normal, adenocarcinoma, lymphoma, GISTs, Crohn's disease, intestinal tuberculosis, Behcet's disease, and cryptogenic multifocal ulcerous stenosing enteritis (CMUSE), were also analyzed.
Finally, the complications of DBE, including abdominal pain, abdominal distension, diarrhea, nausea, perforation, and bleeding, were compared between the adults and elderly.

2.4. Statistical Analysis. SPSS 19.0 was used for data analyses. Continuous variables were expressed as mean \pm SD. Student's $t$-test and Wilcoxon rank sum test were performed for data comparison with and without normal distribution, respectively. A probability $(P)$ value of $<0.05$ was considered statistically significant.

\section{Results}

3.1. Clinical Characteristics of the Adults and Elderly Who Underwent DBE. During the study period, 787 patients were enrolled, $88.6 \%(697 / 787)$ of which received one route of DBE, and $11.4 \%$ (90/787) underwent both routes. A total of 877 DBEs were performed, 729 in the adults and 148 in the elderly. The clinical characteristics of the adults and elderly who underwent DBE are displayed in Table 1. No significant difference in gender and the indications of OGIB and incomplete small bowel obstruction was found between the adults and elderly $(P>0.05)$. In the patients who underwent DBEs, the mean duration of symptoms was approximately 12 weeks $(12.22 \pm 2.89$ and $12.24 \pm 3.10$ weeks, $P=0.944)$. The elderly were more likely to have past GI surgery than the adults; however, no significant difference was found $(P=0.161)$. In the parameters of DBE maneuvering, such as DBE time, route, and examined intestine length, no significant difference was observed between the adults and elderly $(P>0.05)$.

3.2. Diagnostic Yields of DBE between the Adults and Elderly with OGIB. The diagnostic yields of DBE between the adults and elderly with OGIB are listed in Table 2. Among the patients who underwent DBE, approximately $14.3 \%-15.1 \%$ were normal in both groups $(P=0.817)$. The adults had higher tendency to have Meckel's diverticulum than the elderly ( $4.6 \%$ vs. $0.9 \%, P=0.032)$. On the contrary, the elderly were more likely to have angioectasia than the adults $(25.9 \%$ vs. $17.9 \%, P=0.048)$. The two groups were comparable in diagnostic findings, such as adenocarcinoma, lymphoma, GISTs, polyps, Crohn's disease, intestinal tuberculosis, Behcet's disease, and nonspecific enteritis $(P>0.05)$.

3.3. Diagnostic Yields of DBE between the Adults and Elderly with Incomplete Small Bowel Obstruction. The diagnostic yields of DBE between the adults and elderly with incomplete small bowel obstruction are displayed in Table 3. The negative ratios were approximately $11.1 \%-12.8 \%$. The elderly showed a higher frequency of adenocarcinoma than the adults $(19.4 \%$ vs. $7.1 \%, P=0.038)$. However, the adults had a higher frequency of Crohn's disease than the elderly (23.4\% vs. $8.3 \%, P=0.045)$. In terms of lymphoma, GISTs, intestinal tuberculosis, Behcet's disease, and CMUSE, both groups showed comparable ratios $(P>0.05)$.

3.4. Postoperative Complications of DBE in the Adults and Elderly. The postoperative complications of DBE in the adults and elderly are shown in Table 4 . The main postoperative complications of DBE were mild and included 
TABLE 1: Clinical characteristics of adult and elderly patients underwent DBE.

\begin{tabular}{|c|c|c|c|}
\hline & Adults $(n(\%))$ & Elderly $(n(\%))$ & $P$ \\
\hline Total & 729 & 148 & I \\
\hline Gender (M/F) & $401 / 328$ & $82 / 66$ & 0.929 \\
\hline Indications & & & 0.169 \\
\hline Obscure gastrointestinal bleeding & $588(80.7)$ & $112(75.7)$ & \\
\hline Incomplete small bowel obstruction & $141(19.3)$ & $36(24.3)$ & \\
\hline Duration of symptom (week) & $12.22 \pm 2.89$ & $12.24 \pm 3.10$ & 0.944 \\
\hline Past GI surgery & $58(9.9)$ & $17(11.5)$ & 0.161 \\
\hline DBE time (min) & $66.49 \pm 26.10$ & $64.43 \pm 25.37$ & 0.335 \\
\hline DBE route & & & 0.819 \\
\hline Antegrade & $298(40.9)$ & $62(41.9)$ & \\
\hline Retrograde & $431(59.1)$ & $86(58.1)$ & \\
\hline Length of examined intestine $(\mathrm{cm})$ & $130.67 \pm 36.38$ & $129.48 \pm 37.40$ & 0.691 \\
\hline
\end{tabular}

TABLE 2: Diagnostic yields of DBE between adults and elderly patients with OGIB.

\begin{tabular}{lccc}
\hline & $\begin{array}{c}\text { Adults } \mathrm{N}=588 \\
(n(\%))\end{array}$ & $\begin{array}{c}\text { Elderly } \mathrm{N}=112 \\
(n(\%))\end{array}$ & $P$ \\
\hline Normal & $89(15.1)$ & $16(14.3)$ & 0.817 \\
Adenocarcinoma & $33(5.6)$ & $8(7.1)$ & 0.527 \\
Lymphoma & $37(6.3)$ & $7(6.3)$ & 0.986 \\
GISTs & $52(8.8)$ & $9(8.0)$ & 0.781 \\
Meckel's diverticulum & $27(4.6)$ & $1(0.9)$ & 0.032 \\
Polyps & $76(12.9)$ & $15(13.4)$ & 0.893 \\
Crohn's disease & $76(12.9)$ & $11(9.8)$ & 0.361 \\
Intestinal tuberculosis & $28(4.8)$ & $4(3.6)$ & 0.580 \\
Behcet's disease & $27(4.6)$ & $5(4.5)$ & 0.953 \\
Angioectasia & $105(17.9)$ & $29(25.9)$ & 0.048 \\
Nonspecific enteritis & $27(4.6)$ & $4(3.6)$ & 0.736 \\
Others & $11(1.9)$ & $3(2.7)$ & 0.621 \\
\hline
\end{tabular}

TABLE 3: Diagnostic yields of DBE between adults and elderly patients with incomplete small bowel obstruction.

\begin{tabular}{lccc}
\hline & $\begin{array}{c}\text { Adults } \mathrm{N}=141 \\
(n(\%))\end{array}$ & $\begin{array}{c}\text { Elderly } \mathrm{N}=36 \\
(n(\%))\end{array}$ & $P$ \\
\hline Normal & $18(12.8)$ & $4(11.1)$ & 0.786 \\
Adenocarcinoma & $10(7.1)$ & $7(19.4)$ & 0.038 \\
Lymphoma & $15(10.6)$ & $3(8.3)$ & 0.676 \\
GISTs & $9(6.4)$ & $3(8.3)$ & 0.685 \\
Crohn's disease & $33(23.4)$ & $3(8.3)$ & 0.045 \\
Intestinal tuberculosis & $15(10.6)$ & $6(16.7)$ & 0.336 \\
Behcet's disease & $13(9.2)$ & $4(11.1)$ & 0.735 \\
CMUSE & $22(15.6)$ & $4(11.1)$ & 0.497 \\
Others & $6(4.3)$ & $2(5.6)$ & 0.744 \\
\hline
\end{tabular}

abdominal pain, abdominal distension, diarrhea, and nausea. The adults and elderly displayed comparable complications $(P>0.05)$. Postoperative bleeding occurred in two patients,
TABle 4: Postoperative complications of DBE in adults and elderly patients.

\begin{tabular}{lccc}
\hline & Adults $(n(\%))$ & Elderly $(n(\%))$ & $P$ \\
\hline Abdominal pain & $19(2.6)$ & $6(4.1)$ & 0.357 \\
Abdominal distention & $17(2.3)$ & $6(4.1)$ & 0.260 \\
Diarrhea & $22(3.0)$ & $4(6.8)$ & 0.835 \\
Nausea & $28(3.8)$ & $7(4.7)$ & 0.615 \\
Bleeding & $2(0.3)$ & $0(0.0)$ & 0.426 \\
Perforation & $0(0.0)$ & $1(0.7)$ & 0.169 \\
\hline
\end{tabular}

with no significant difference between the adults and elderly $(P=0.426)$. Only 1 patient in both groups suffered from perforation, a severe complication $(P=0.169)$.

\section{Discussions}

The advent of the aging society in China has brought great concerns on elderly health care. Elderly patients have distinct disease spectrum and well-being. The diagnostic yield and tolerability of DBE in elderly patients compared with adults should be determined.

Given its technical complexity and potential invasiveness, DBE is not always the first choice in detecting small bowel lesions. The diagnostic efficacy and yield of DBE have become a major concern for physicians. In our previous findings, OGIB and incomplete small bowel obstruction have the highest (approximately 80\%) diagnostic yields compared with other indications [3]. This finding prompted us to further discover the discordance of diagnostic yields between adults and elderly. This study retrospectively reviewed 877 DBEs within 10 years in our hospital. We believe that this population is a fairly large cohort that may represent the characteristics and outcomes of Chinese adults and elderly patients receiving DBEs.

This study showed that over 10 years, the overall diagnostic yields of DBE in OGIB or incomplete small bowel obstruction had increased to $84.9 \%-88.9 \%$, which is much higher than our previous findings [3] and those of a recent study 
in China by Wang et al. [17]. This result might be ascribed to the optimization of DBE indication and the improved guidance of other diagnostic modalities, such as CT enterography and MR enterography. For patients with OGIB, Meckel's diverticulum was more observed in the adults than in the elderly and the reverse in angioectasia. These findings further indicated that the proportion of lesions in adults and elderly had some difference. In patients with incomplete small bowel obstruction, Crohn's disease was more prevalent in the adults than in the elderly, which may be due to the high incidence of Crohn's disease in young patients [18]. On the contrary, the elderly had a higher incidence of adenocarcinoma than the adults. This finding might be ascribed to the high chance of malignancy in the elderly [19]. Our findings were similar to those of other studies $[13,15,20,21]$.

Despite the usefulness of DBE in diagnosing small bowel disease, its safety should be analyzed in the elderly. Furthermore, approximately $12.0 \%-20.4 \%$ of the patients underwent discomfort postoperatively. Most of the discomforts were mild and could be successfully alleviated through symptomatic treatment. The complication rate was higher than that observed in other researches $[12-14,16]$. A possible reason is that many patients did not receive midazolam and fentanyl for sedation, for they had to change body positions frequently to facilitate deeper insertion. These medicines are generally administered to improve patient cooperation and ameliorate discomfort. No significant difference in complication was found between the adults and elderly, further proving the safety of DBE in the elderly. Only one patient experienced perforation after the procedure, which might be ascribed to the multiple operations and colostomy he received. This finding suggests the careful performance of DBE in patients with postoperative ankylenteron or surgically altered anatomy.

Our study has several limitations. First, this study was designed retrospectively. Second, we defined elderly as individuals aged $\geq 65$ years and did not subdivide the elderly into additional groups for evaluation. In the future, we could carry out additional prospective studies with much old patients enrolled to further analyze the feasibility and safety of DBE in the extremely old population.

In conclusion, DBE has high a diagnostic yield in small bowel disorders with slightly different disease spectrum between the adults and elderly. Most of the postoperative complications of DBE is mild and could be properly alleviated. The tolerance of the elderly to $\mathrm{DBE}$ is comparable to that of the adults.

\section{Data Availability}

Data was available through contacting Prof. Jie Zhong using email.

\section{Ethical Approval}

The institutional ethics board of Ruijin Hospital approved our research.

\section{Conflicts of Interest}

All the authors had no conflict of interest and approved this article for submission.

\section{Authors' Contributions}

Lei Wang and Mengfan Xie contributed equally to this work.

\section{Acknowledgments}

This study was supported by the National Natural Science Foundation of China (Nos. 81670503, 81970489), Shanghai Committee of Science and Technology Foundation (No. 16411950402) and Shanghai Health and Family Planning Committee (No. 201640112).

\section{References}

[1] H. Yamamoto, H. Kita, K. Sunada et al., "Clinical outcomes of double-balloon endoscopy for the diagnosis and treatment of small-intestinal diseases," Clinical Gastroenterology and Hepatology, vol. 2, no. 11, pp. 1010-1016, 2004.

[2] J. S. Byeon, J. W. Chung, K. D. Choi et al., "Clinical features predicting the detection of abnormalities by double balloon endoscopy in patients with suspected small bowel bleeding," Gastroenterología y Hepatología, vol. 23, no. 7part1, pp. 1051-1055, 2008.

[3] J. Zhong, T. Ma, C. Zhang et al., "A retrospective study of the application on double-balloon enteroscopy in 378 patients with suspected small-bowel diseases," Endoscopy, vol. 39, no. 3, pp. 208-215, 2007.

[4] L. Aabakken, M. Bretthauer, and P. D. Line, "Double-balloon enteroscopy for endoscopic retrograde cholangiography in patients with a Roux-en-Y anastomosis," Endoscopy, vol. 39, no. 12, pp. 1068-1071, 2007.

[5] D. S. Emmett and D. B. Mallat, "Double-balloon ERCP in patients who have undergone Roux-en- $Y$ surgery: a case series," Gastrointestinal Endoscopy, vol. 66, no. 5, pp. 10381041, 2007.

[6] D. H. Kim, J. S. Byeon, S. K. Lee et al., "Usefulness of double balloon endoscopy in patients with surgically distorted intestinal anatomy," Journal of Clinical Gastroenterology, vol. 43, no. 8, pp. 737-742, 2009.

[7] G. D. N. Heine, M. Hadithi, M. J. M. Groenen, E. J. Kuipers, M. A. Jacobs, and C. J. Mulder, "Double-balloon enteroscopy: indications, diagnostic yield, and complications in a series of 275 patients with suspected small-bowel disease," Endoscopy, vol. 38, no. 1, pp. 42-48, 2006.

[8] P. B. F. Mensink, J. Haringsma, T. Kucharzik et al., "Complications of double balloon enteroscopy: a multicenter survey," Endoscopy, vol. 39, no. 7, pp. 613-615, 2007.

[9] A. May, L. Nachbar, J. Pohl, and C. Ell, "Endoscopic interventions in the small bowel using double balloon enteroscopy: feasibility and limitations," The American Journal of Gastroenterology, vol. 102, no. 3, pp. 527-535, 2007.

[10] L. B. Gerson, J. L. Fidler, D. R. Cave, and J. A. Leighton, "ACG clinical guideline: diagnosis and management of small bowel bleeding," The American Journal of Gastroenterology, vol. 110, no. 9, pp. 1265-1287, 2015. 
[11] S. Zepeda-Gómez, R. Barreto-Zuñiga, S. Ponce-de-León et al., "Risk of hyperamylasemia and acute pancreatitis after doubleballoon enteroscopy: a prospective study," Endoscopy, vol. 43, no. 9, pp. 766-770, 2011.

[12] O. Möschler, A. May, M. K. Müller, and C. Ell, “Complications in and performance of double-balloon enteroscopy (DBE): results from a large prospective DBE database in Germany," Endoscopy, vol. 43, no. 6, pp. 484-489, 2011.

[13] L. Xin, Z. Liao, Y. P. Jiang, and Z. S. Li, "Indications, detectability, positive findings, total enteroscopy, and complications of diagnostic double-balloon endoscopy: a systematic review of data over the first decade of use," Gastrointestinal Endoscopy, vol. 74, no. 3, pp. 563-570, 2011.

[14] S. R. Hegde, K. Iffrig, T. Li et al., "Double-balloon enteroscopy in the elderly: safety, findings, and diagnostic and therapeutic success," Gastrointestinal Endoscopy, vol. 71, no. 6, pp. 983989, 2010.

[15] Q. He, Q. Zhang, J. D. Li et al., "Double balloon enteroscopy in the old: experience from China," World Journal of Gastroenterology, vol. 18, no. 22, pp. 2859-2866, 2012.

[16] J. S. Byeon, N. K. Mann, L. H. Jamil, and S. K. Lo, "Double balloon enteroscopy can be safely done in elderly patients with significant co-morbidities," Journal of Gastroenterology and Hepatology, vol. 27, no. 12, pp. 1831-1836, 2012.

[17] P. Wang, Y. Wang, Y. Dong et al., "Outcomes and safety of double-balloon enteroscopy in small bowel diseases: a singlecenter experience of 1531 procedures," Surgical Endoscopy, vol. 18, 2020.

[18] L. Prideaux, M. A. Kamm, P. P. de Cruz, F. K. L. Chan, and S. C. Ng, "Inflammatory bowel disease in Asia: a systematic review," Journal of Gastroenterology and Hepatology, vol. 27, no. 8, pp. 1266-1280, 2012.

[19] D. J. Cangemi, M. K. Patel, V. Gomez, J. R. Cangemi, M. E. Stark, and F. J. Lukens, "Small bowel tumors discovered during double-balloon enteroscopy: analysis of a large prospectively collected single-center database," Journal of Clinical Gastroenterology, vol. 47, no. 9, pp. 769-772, 2013.

[20] S. R. Jeon, J. O. Kim, H. G. Kim et al., "Changes over time in indications, diagnostic yield, and clinical effects of doubleballoon enteroscopy," Clinical Gastroenterology and Hepatology, vol. 10, no. 10, pp. 1152-1156, 2012.

[21] C. C. Hansen and K. Søreide, "Systematic review of epidemiology, presentation, and management of Meckel's diverticulum in the 21st century," Medicine, vol. 97, no. 35, article e12154, 2018. 